\title{
HOW TO OBTAIN AN ASYMPTOTIC EXPANSION OF A SEQUENCE FROM AN ANALYTIC IDENTITY SATISFIED BY ITS GENERATING FUNCTION
}

\section{J. M. PLOTKIN and JOHN W. ROSENTHAL}

(Received 22 February 1990; revised 30 October 1991)

Communicated by Louis Caccetta

\begin{abstract}
Let $f_{n}$ be a sequence of nonnegative integers and let $f(x):=\sum_{n \geq 0} f_{n} x^{n}$ be its generating function. Assume $f(x)$ has the following properties: it has radius of convergence $r, 0<r<1$, with its only singularity on the circle of convergence at $x=r$ and $f(r)=s ; y=f(x)$ satisfies an analytic identity $F(x, y)=0$ near $(r, s)$; for some $k \geq 2 F_{0, j}=0,0 \leq j<k, F_{0, k} \neq 0$ where $F_{i, j}$ is the value at $(r, s)$ of the $i^{\text {th }}$ partial derivative with respect to $x$ and the $j^{\text {th }}$ partial derivative with respect to $y$ of $F$. These assumptions form the basis of what we call the typical and general cases. In both cases we show how to obtain an asymptotic expansion of $f_{n}$. We apply our technique to produce several terms in the asymptotic expansion of combinatorial sequences for which previously only the first term was known.
\end{abstract}

1991 Mathematics subject classification (Amer. Math. Soc.): primary 05 A 15 ; secondary 05 C 30.

Keywords and phrases: Generating function, analytic identity, Weierstrass Preparation Theorem, fractional power series, asymptotic expansion, trees, number of trees.

\section{Introduction}

Pólya [8] developed a technique to use an analytic identity satisfied by the generating function of a sequence (arising in combinatorics) to asymptotically determine the terms in the sequence. This technique was further developed and studied by many authors including $[1,3,5,6]$. It involves the following two situations:

(C) 1994 Australian Mathematical Society 0263-6115/94 \$A2.00+0.00 


\section{Situation 1}

Let $f_{n}$ be a sequence of nonnegative integers arising in combinatorics. Let $f(x):=\sum_{n \geq 0} f_{n} x^{n}$ be its generating function, viewed as a function of a complex variable. Let $r$ be its radius of convergence.

ASSUMPTION 1. The radius of convergence $r$ satisfies $0<r<1$ and $x=r$ is the only singularity of $f$ on its circle of convergence.

ASSUMPTION 2. The function $f(r)$ converges, say to $s$.

AsSUMPTION 3. There is a function $F(x, y)$, which is analytic in a neighborhood of $(r, s)$, such that $x$ close to and less than or equal to $r$ implies $F(x, f(x))=0$.

NOTATiON. If $G(x, y)$ is a function, then $G_{i, j}$ is the value at $(r, s)$ of the $i^{t h}$ partial derivatives with respect to $x$ of the $j^{\text {th }}$ partial derivative with respect to $y$ of $G$.

ASSUMPTION 4. For some $k \geq 2, F_{0, j}=0$ for $0 \leq j<k$ and $F_{0, k} \neq 0$.

In most cases in the literature $k=2$ in Assumption 4 and furthermore

ASSUMPTION 5. That $F_{1,0} \neq 0$.

DEFINITION. The typical case consists of Assumptions 1 through 5 with $k=2$ in Assumption 4. The general case consists of Assumptions 1 through 4 and Assumptions 6 through 9 (presented in Section 2).

REMARK. Assumptions 6 through 9 hold in the typical case.

\section{Situation 2}

Let $f_{n}$ be a sequence of nonnegative integers arising in combinatorics. Let $f(x)$ be its generating function. Assume there is a sequence $g_{n}$ with generating function $g(x)$ such that the general case holds for $g_{n}$ and $f(x)$ differs from a polynomial in $x$ and $g(x)$ by a function with a radius of convergence greater than that of $g(x)$.

In this paper we are interested in obtaining in both situations not only an asymptotic formula for $f_{n}$ (as in the previously cited works) but also an asymptotic expansion of $f_{n}$. In Section 2 we show how to obtain such an expansion 
in the general case (of Situation 1) giving special emphasis to the typical case. We also observe how the work used to obtain asymptotic expansions in Situation 1 can also often be used to obtain asymptotic expansions in Situation 2. In Section 3 we apply these results to obtain the first few terms in the asymptotic expansion of combinatorial sequences for which previously only the first term was known. In particular we study several sequences that arise in [7], and in counting various types of trees studied in [5] and [3]. We correct errors in many of the constants obtained in [3].

\section{Abstract discussion of the two situations}

2.1. Situation 1 To obtain the asymptotic expansion we show: (1) how to convert the analytic identity into a 'polynomial' identity; (2) how to use the 'polynomial' identity to obtain a fractional power series expansion of $f(x)$ about $r$; (3) how to use the fractional power series expansion to obtain an asymptotic expansion of $f_{n}$ in terms of $\left(\begin{array}{c}a+k b \\ n\end{array}\right)$ 's, where $k$ is a nonnegative integer and $a$ and $b$ are rational numbers; and (4) how to convert this asymptotic expansion into an asymptotic expansion in terms of powers of $n$.

Step 1 By the Weierstrass Preparation Theorem (see, for example, [4]) Assumption 4 implies that in a neighborhood of $(r, s)$

$$
F(x, y)=A(x, y) \cdot P(x, y),
$$

where $A(x, y)$ is analytic, $A(r, s) \neq 0$, and

$$
P(x, y)=(y-s)^{k}+\sum_{j=0}^{k-1} p_{j}(x)(y-s)^{j}
$$

where the $p_{i}(x)$ are analytic and vanish at $x=r$. It may not be possible to determine the polynomial $P$. Instead we can determine the partial derivatives $P_{i, j}$. These and the partial derivatives $A_{i, j}$ are recursively obtained from the $F_{i, j}$ 's as follows:

By definition $P_{0, j}=0$ for $j \neq k$ and $P_{0, k}=k !$.

By the product rule

$$
F_{i, j}=\sum_{p=0}^{i} \sum_{q=0}^{j}\left(\begin{array}{c}
i \\
p
\end{array}\right)\left(\begin{array}{c}
j \\
q
\end{array}\right) A_{p, q} P_{i-p, j-q} .
$$


So for $j<k$

$$
F_{i, j}=\sum_{p=1}^{i-1} \sum_{q=0}^{j}\left(\begin{array}{c}
i \\
p
\end{array}\right)\left(\begin{array}{l}
j \\
q
\end{array}\right) A_{p, q} P_{i-p, j-q}+\sum_{q=1}^{j}\left(\begin{array}{c}
j \\
q
\end{array}\right) A_{0, q} P_{i, j-q}+A_{0,0} P_{i, j}
$$

and hence

$P_{i, j}=\left[F_{i, j}-\sum_{p=1}^{i-1} \sum_{q=0}^{j}\left(\begin{array}{c}i \\ p\end{array}\right)\left(\begin{array}{l}j \\ q\end{array}\right) A_{p, q} P_{i-p, j-q}-\sum_{q=1}^{j}\left(\begin{array}{c}j \\ q\end{array}\right) A_{0, q} P_{i, j-q}\right] / A_{0,0}$.

And for $j \geq k$,

$$
F_{i, j}=\sum_{p=0}^{i-1} \sum_{q=0}^{j}\left(\begin{array}{c}
i \\
p
\end{array}\right)\left(\begin{array}{c}
j \\
q
\end{array}\right) A_{p, q} P_{i-p, j-q}+\left(\begin{array}{c}
j \\
k
\end{array}\right) k ! A_{i, j-k} .
$$

Hence, (replacing $j-k$ by $j$ )

(2) $A_{i, j}=\left[F_{i, j+k}=\sum_{p=0}^{i-1} \sum_{q=0}^{j+k}\left(\begin{array}{c}i \\ p\end{array}\right)\left(\begin{array}{c}j+k \\ q\end{array}\right) A_{p, q} P_{i-p, j+k-q}\right] \frac{j !}{(j+k) !}$.

By induction on $j+i k$ Formulae (1) and (2) express the $A_{i, j}$ 's and the $P_{i, j}$ 's in terms of $F_{p, q}$ 's for $p \leq i$ and $q \leq j+i k$.

For each $j$ from 0 to $k-1, p_{j}(x)$ can be written as $\sum_{i \geq i_{j}} p_{i, j}(1-x / r)^{i}$, where $i_{j}$ is the order of the zero of $p_{j}(x)$ at $x=r$ (or $+\infty$ if $p_{j}(x)$ is identically zero).

An easy computation shows

$$
p_{i, j}=\frac{P_{i, j}(-r)^{i}}{i ! j !} \quad \text { for } \quad 0 \leq j \leq k-1 \quad \text { and } \quad i \geq i_{j} .
$$

Also (as observed in [6]) for $0 \leq j \leq k-1, i_{j}$ is the least $i$ such that $F_{i, j} \neq 0$.

Step 2 By a classical result (for example, see Walker [10, Chapter 4, Section 3]), the 'polynomial' identity $P(x, f(x))=0$ for $x$ close to and less than or equal to $r$ implies that $f(x)$ can be expressed as a fractional power series $\sum_{i=1}^{\infty} a_{i}(1-x / r)^{s_{i}}$ or $\sum_{i=1}^{m} a_{i}(1-x / r)^{s_{i}}$, where $s_{i}$ is an increasing sequence of rational numbers, and $a_{i} \neq 0$. As $f(r)$ converges, $s_{1} \geq 0$.

We now observe how under the weak technical Assumptions 6, 7, 8, and 9 the algorithm in Walker can be used to express the $a_{i}$ 's and the $s_{i}$ 's in terms of the $p_{i, j}$ 's of Step 1 . 
ASSUMPTION 6. That $i_{0} / k$ is not an integer.

ASSUMPTION 7. That $i_{j} \geq i_{0}(1-j / k)$ for $1 \leq j \leq k-1$.

In the typical case these assumptions hold as $k=2$ and $i_{0}=1$. As observed in [6] Assumptions 6 and 7 imply $s_{1}=i_{0} / k$ and $g\left(a_{1}\right)=0$, where

$$
g(a)=a^{k}+\sum\left\{p_{i_{j}, j} a^{j}: 1 \leq j \leq k-1 \text { and } i_{j}=s_{1}(k-j)\right\}+p_{i_{0}, 0}
$$

ASSUMPTION 8 . That $g(a)=0$ has only one solution for which $a / \Gamma\left(-s_{1}\right)$ is a positive real (where $\Gamma$ is the classical gamma function).

We choose $a_{1}$ to be this solution. Assumption 8 is used in Step 3 .

ASSUMPTION 9. That $a_{1}$ is not a multiple root of $g(a)=0$.

In the typical case as observed in Bender [1] $g(a)=a^{2}+p_{1,0}$ and $p_{1,0} \neq 0$ and, hence, Assumptions 8 and 9 hold.

Assumption 9 tells us that the $r$ of Walker [10, p. 100] equals 1 and hence $a_{i}$ and $s_{i}$ for $i \geq 2$ can be determined by the technique suggested in Walker [10, Section 3.3]. For simplicity we illustrate this in the typical case.

Say $P(x, y)=(y-s)^{2}+p(x)(y-s)+q(x)$, where

$$
p(x)=\sum_{i \geq 1} p_{i}(1-x / r)^{i} \quad \text { and } \quad q(x)=\sum_{i \geq 1} q_{i}(1-x / r)^{i} .
$$

The fractional power series of $f(x)-s$ has the form

$$
\sum_{i \geq 0} b_{i}(1-x / r)^{i+1 / 2}+\sum_{i \geq 1} c_{i}(1-x / r)^{i} .
$$

Equating coefficients of the various powers of $(1-x / r)^{i}$ in $P(x, f(x))$, we obtain:

(4) $2 \sum\left\{b_{i} c_{j}: i+j=m\right.$ and $\left.j \geq 1\right\}+\sum\left\{b_{i} p_{j}: i+j=m\right.$ and $\left.j \geq 1\right\}=0$ for $m \geq 1$ (using the half integer powers);

$$
\left(b_{0}\right)^{2}+q_{1}=0
$$

(using the lowest integer power); and

$$
\begin{aligned}
\sum\left\{b_{i} b_{j}: i+j=\right. & m-1\}+\sum\left\{c_{i} c_{j}: i+j=m, i \geq 1 \text { and } j \geq 1\right\} \\
& +\sum\left\{p_{i} c_{j}: i+j=m, i \geq 1 \text { and } j \geq 1\right\}+q_{m}=0
\end{aligned}
$$


for $m \geq 1$ (using the remaining integer powers).

By induction and (4), $c_{i}=-p_{i} / 2$. So (6) gives

$\sum\left\{b_{i} b_{j}: i+j=m-1\right\}-1 / 4 \sum\left\{p_{i} p_{j}: i+j=m, i \geq 1\right.$ and $\left.j \geq 1\right\}+q_{m}=0$ and, hence, (replacing $m-1$ by $m$ )

(7) $b_{m}=\left[-\sum\left\{b_{i} b_{j}: i+j=m, i \geq 1\right.\right.$ and $\left.j \geq 1\right\}$ $+1 / 4 \sum\left\{p_{i} p_{j}: i+j=m+1, i \geq 1\right.$ and $\left.\left.j \geq 1\right\}-q_{m+1}\right] / 2 b_{0}$.

By (5), $b_{0}=-\sqrt{-q_{1}}$. So using (7) by induction $b_{m}$ may be expressed in terms of the $p_{i}$ 's and the $q_{i}$ 's for $i \leq m+1$.

REMARK. Even if one does not make Assumptions 6 through 9, it may still be possible to use the techniques in Walker to express the coefficients of the fractional power series of $f(x)$ in terms of the $p_{i, j}$ 's. (In [6] we showed how to obtain the first noninteger power term and its coefficient.) The only difficulty is that one may obtain several possible fractional power series. In this case one needs some procedure to determine which of these is the correct one.

Step 3 Next we use the following special case of a theorem of Darboux (see [9], for example).

THEOREM. Let $f_{n}$ be a sequence of nonnegative integers with generating function $f(x)$ satisfying Assumptions 1 and 2 . If for $x$ near $r, f(x)$ is expressible as the fractional power series $\sum_{i>0} a_{i}(1-x / r)^{(a+i b)}$, where $a$ and $b$ are rational numbers and $b>0$, then $f_{n}$ has the asymptotic expansion

$$
\sum_{i \geq 0} a_{i}\left(\begin{array}{c}
a+i b \\
n
\end{array}\right)(-1)^{n} r^{-n}
$$

Using this theorem we can obtain an asymptotic expansion of $f_{n}$ in terms of the numbers $\left(\begin{array}{c}a+i b \\ n\end{array}\right)$.

In the typical case we use this theorem for $a=0$ and $b=1 / 2$. In this case it may be stated as follows:

THEOREM. Let $f_{n}$ be a sequence of nonnegative integers with generating function $f(x)$ satisfying Assumptions 1 and 2 . If for $x$ near $r, f(x)$ is expressible as the fractional power series $\sum_{i \geq 0} b_{i}(1-x / r)^{i+1 / 2}+\sum_{i \geq 0} c_{i}(1-x / r)^{i}$; 
then $f_{n}$ has the asymptotic expansion

$$
\sum_{i \geq 0} b_{i}\left(\begin{array}{c}
i+1 / 2 \\
n
\end{array}\right)(-1)^{n} r^{-n}
$$

Step 4 To obtain an asymptotic expansion of $f_{n}$ in terms of powers of $n$ it remains to obtain such asymptotic expansions of $\left(\begin{array}{c}a+i b \\ n\end{array}\right)$. This may be done by using the following asymptotic expansion form of Stirling's Formula:

$$
\ln \Gamma(z)=(z-1 / 2) \ln (z)-z+1 / 2 \ln (2 \pi)+\sum_{t=1}^{\infty} \frac{(-1)^{t-1} B_{t}}{2 t(t-1) z^{2 t-1}},
$$

where $B_{t}$ is the $t^{\text {th }}$ Bernouilli number.

Specifically we note

$$
\left(\begin{array}{l}
\alpha \\
n
\end{array}\right)=\frac{\Gamma(n-\alpha)}{\Gamma(n+1)} \cdot \frac{1}{\Gamma(-\alpha)} \cdot(-1)^{n}
$$

and apply Stirling's Formula to $\Gamma(n-\alpha)$ and $\Gamma(n+1)$. For the typical case (obtained with the assistance of MACSYMA):

$$
\begin{aligned}
& \left(\begin{array}{c}
1 / 2 \\
n
\end{array}\right)=-\left(1+\frac{3}{8 n}+\frac{25}{128 n^{2}}+\frac{105}{1024 n^{3}}+\frac{1659}{32768 n^{4}}+\ldots\right) \frac{(-1)^{n}}{n^{3 / 2}} \frac{1}{2 \sqrt{\pi}} \\
& \left(\begin{array}{c}
3 / 2 \\
n
\end{array}\right)=-\left(1+\frac{15}{8 n}+\frac{385}{128 n^{2}}+\frac{4725}{1024 n^{3}}+\ldots\right) \frac{(-1)^{n}}{n^{5 / 2}} \frac{3}{4 \sqrt{\pi}} \\
& \left(\begin{array}{c}
5 / 2 \\
n
\end{array}\right)=-\left(1+\frac{35}{8 n}+\frac{1785}{128 n^{2}}+\ldots\right) \frac{(-1)^{n}}{n^{7 / 2}} \frac{15}{8 \sqrt{\pi}} \\
& \left(\begin{array}{c}
7 / 2 \\
n
\end{array}\right)=-\left(1+\frac{63}{8 n}+\ldots\right) \frac{(-1)^{n}}{n^{9 / 2}} \frac{105}{16 \sqrt{\pi}} \\
& \left(\begin{array}{c}
9 / 2 \\
n
\end{array}\right)=-(1+\ldots) \frac{(-1)^{n}}{n^{11 / 2}} \frac{945}{32 \sqrt{\pi}}
\end{aligned}
$$

\section{Conclusions in the typical case}

In the typical case the four steps imply that $f_{n}$ has the asymptotic expansion

$$
\left(1 /\left(2 \sqrt{\pi} r^{n} n^{3 / 2}\right)\right)\left(\sum_{i \geq 0} A_{i} / n^{i}\right), \text { where } A_{0}=\sqrt{\left(2 F_{1,0} r / F_{0,2}\right)} \text { (as in [1]) }
$$

and

$$
\begin{aligned}
A_{1}=[ & \left\{9\left(F_{0,2}\right)^{3} \dot{F}_{2,0}-18\left(F_{0,2}\right)^{2} F_{1,0} F_{1,2}-9\left(F_{0,2}\right)^{2}\left(F_{1,1}\right)^{2}+18 F_{0,2} F_{0,3} F_{1,0} F_{1,1}\right. \\
& \left.\left.+3 F_{0,2} F_{0,4}\left(F_{1,0}\right)^{2}-5\left(F_{0,2}\right)^{3}\left(F_{1,0}\right)^{2}\right\} r^{2}+9\left(F_{0,2}\right)^{3} F_{1,0} r\right] /\left[12\left(F_{0,2}\right)^{4} A_{0}\right]
\end{aligned}
$$


Each $A_{i}$ may be expressed in terms of partial derivatives of $F$. These expressions for $A_{i}$ grow rapidly in size as $i$ increases. They are not worthy of display and should only be computed using computer algebra. Nonetheless the results of such computations can be efficiently used with further computer algebra assistance to determine several terms of the asymptotic expansion of particular sequences of combinatorial interest. This is done throughout Section 3.

2.2. Situation 2 Say $f(x)=Q(x, g(x))+h(x)$, where $f(x)$ is the generating function of a sequence $f_{n}, Q$ is a polynomial in both its variables, $g(x)$ is the generating function of a general case sequence $g_{n}$, and $h(x)$ has a radius of convergence greater than $r$, the radius of convergence of $g(x)$.

By Steps 1 and 2 for $g_{n}, g(x)$ has a fractional power series about $x=r$. In many cases plugging this fractional power series in for $g(x)$ in $Q(x, g(x))$ gives a fractional power series which has some noninteger powers of $(1-x / r)$. As $h(x)$ is analytic at $x=r$, applying Steps 3 and 4 to this new fractional power series gives an asymptotic expansion of $f_{n}$.

\section{Some sequences of combinatorial interest}

All sequences considered in this section are ones in the typical case for which the first order term in the asymptotic expansion is already known.

3.1. Several sequences from [6] In [6] we introduced several sequences including $\sharp_{n}, u_{n}$, and $m_{n}$ in a study of the expected complexity of an analytic tableaux algorithm for the satisfiability problem of propositional calculus. That paper shows that the generating functions satisfy the identities:

$$
\begin{aligned}
\sharp(x) & =1+2 x(\sharp(x))^{2} ; \\
u(x) & =1+2 x \sharp(x) u(x)+x(u(x))^{2} ; \quad \text { and } \\
m(x) & =1+2 x \sharp(x) m(x)+2 x(u(x))^{2}+x(m(x))^{2} .
\end{aligned}
$$

It obtains the first order terms of an asymptotic expansion of each of these sequences.

Also we observed that $\sharp_{n}=2^{n} c_{n}$, where $c_{n}$ is the $n^{\text {th }}$ Catalan number. As is well known, $c_{n}=(1 /(2 n+1))\left(\begin{array}{c}2 n+1 \\ n\end{array}\right)$ and, hence, asymptotic expansions of $c_{n}$ and $\sharp_{n}$ can be determined by using the asymptotic expansion version of Stirling's Formula. Alternately the same asymptotic expansions can be obtained using Steps 2 through 4 . Similarly asymptotic expansions of $u_{n}$ and $m_{n}$ can be obtained using these steps. With computer algebra we obtained. 
THEOREM 1. (a) $\sharp_{n}$ has the asymptotic expansion

$$
\frac{8^{n} n^{-3 / 2}}{\sqrt{\pi}}\left(1-\frac{9}{8 n}+\frac{145}{128 n^{2}}-\frac{1155}{1024 n^{3}}+\frac{36939}{32768 n^{4}}+\ldots\right) ;
$$

(b) $u_{n}$ has the asymptotic expansion

$$
\begin{aligned}
9^{n} n^{-3 / 2}\left(1.465807536-\frac{0.5496778259}{n}\right. & +\frac{11.27984705}{n^{2}} \\
& \left.+\frac{182.6175746}{n^{3}}+\frac{4914.966960}{n^{4}}+\ldots\right) ;
\end{aligned}
$$

(c) $m_{n}$ has the asymptotic expansion

$$
\begin{aligned}
\mu^{-n} n^{-3 / 2}\left(2.020870239+\frac{0.1180427831}{n}\right. & +\frac{16.74728853}{n^{2}} \\
& \left.+\frac{194.4880010}{n^{3}}+\frac{3562.577082}{n^{4}}+\ldots\right),
\end{aligned}
$$

where $\mu=(-13+44 \sqrt{2}) / 529$.

It is interesting to see in each case how the higher order terms affect the accuracy of estimates.

\begin{tabular}{c|c|lllll} 
& Precise & \multicolumn{4}{|c}{ Approximations (rounded to nearest integer) } \\
\cline { 3 - 7 } value & First & Second & Third & Fourth & Fifth \\
of $\sharp_{n}$ & order & order & order & order & order \\
\hline 10 & 17199104 & 19156887 & 17001737 & 17218749 & 17197141 & 17199301
\end{tabular}

For $\sharp_{n}$ at $n=100$ each of the first five orders of approximation increases the accuracy by about two decimal places. (Intuitively we should expect this as the coefficients obtained in the expansion are all roughly the same).

\begin{tabular}{c|c|cccll} 
& Precise & \multicolumn{4}{|c}{ Approximations (rounded to nearest integer) } \\
\cline { 3 - 7 } $\mathrm{n}$ & $\begin{array}{l}\text { value } \\
\text { of } u_{n}\end{array}$ & $\begin{array}{l}\text { First } \\
\text { order }\end{array}$ & $\begin{array}{l}\text { Second } \\
\text { order }\end{array}$ & $\begin{array}{l}\text { Third } \\
\text { order }\end{array}$ & $\begin{array}{l}\text { Fourth } \\
\text { order }\end{array}$ & $\begin{array}{l}\text { Fifth } \\
\text { order }\end{array}$ \\
\hline 10 & 135733168 & 161622583 & 155561737 & 167999099 & 188134843 & 242328155
\end{tabular}

Intuitively the accuracy is so poor because the coefficients increase so rapidly. On the other hand by $n=70$, the fifth order approximation is accurate to 4 places and is better than any of the first through fourth order approximations.

The numerical evidence for the accuracy of the approximations of $m_{n}$ is quite similar to that for $u_{n}$. 
3.2. Trees Pólya [8] and Otter [5] studied the number of trees. (See also [1]).

Let $b_{n}$ be the number of planted unlabelled binary trees with $n$ terminal nodes; let $t_{n}$ be the number of unlabelled trees with $n$ vertices; and let $r_{n}$ be the number of rooted unlabelled trees with $n$ vertices.

The above authors derived the following generating function identities:

$$
\begin{aligned}
& \left.b(x)=x+1 / 2\left((b(x))^{2}+b\left(x^{2}\right)\right)\right) ; \\
& r(x)=x \exp \left(\sum_{n \geq 1} r\left(x^{n}\right) / n\right) ; \quad \text { and } \\
& t(x)=r(x)-1 / 2\left((r(x))^{2}-r\left(x^{2}\right)\right) .
\end{aligned}
$$

They also determined the radii of convergence of the generating functions and the first order terms of asymptotic expansions. Using Steps 2 through 4 for $b_{n}$, Steps 1 through 4 for $r_{n}$, and the procedure in Section 2.2 for $t_{n}$, we obtained with computer algebra:

THEOREM 2. (a) $b_{n}$ has the asymptotic expansion

$$
\beta^{n} n^{-3 / 2}\left(0.3187766259+\frac{0.2038317427}{n}+\frac{0.3682702316}{n^{2}}+\frac{1.476819367}{n^{3}}+\ldots\right) \text {, }
$$

where $\beta=0.4026975037$.

(b) $r_{n}$ has the asymptotic expansion

$$
\rho^{-n} n^{-3 / 2}\left(0.4399240126+\frac{0.04416990184}{n}+\frac{0.2216928060}{n^{2}}+\frac{0.8676554908}{n^{3}}+\ldots\right),
$$

and $t_{n}$ has the asymptotic expansion

\begin{tabular}{|c|c|c|c|c|c|}
\hline \multirow[b]{2}{*}{$n$} & \multirow{2}{*}{$\begin{array}{c}\text { Precise } \\
\text { value } \\
\text { of } b_{n}\end{array}$} & \multicolumn{4}{|c|}{ Approximations (rounded to nearest integer) } \\
\hline & & $\begin{array}{l}\text { First } \\
\text { order }\end{array}$ & $\begin{array}{l}\text { Second } \\
\text { order }\end{array}$ & $\begin{array}{l}\text { Third } \\
\text { order }\end{array}$ & $\begin{array}{l}\text { Fourth } \\
\text { order }\end{array}$ \\
\hline 20 & 293547 & 283376 & 292436 & 293254 & 293418 \\
\hline & Precise & \multicolumn{4}{|c|}{ Approximations (rounded to nearest integer) } \\
\hline $\mathrm{n}$ & $\begin{array}{l}\text { value } \\
\text { of } r_{n}\end{array}$ & $\begin{array}{l}\text { First } \\
\text { order }\end{array}$ & $\begin{array}{l}\text { Second } \\
\text { order }\end{array}$ & $\begin{array}{l}\text { Thir } \\
\text { orde }\end{array}$ & $\begin{array}{l}\text { Fourth } \\
\text { order }\end{array}$ \\
\hline 18 & 1721159 & 1708154 & 1717682 & 1720 & 1720916 \\
\hline
\end{tabular}

$$
\rho^{-n} n^{-5 / 2}\left(0.5349496061+\frac{0.4853877311}{n}+\frac{2.379745574}{n^{2}}+\ldots\right),
$$

where $\rho=0.3383218569$.

Here is some numerical evidence of the improvement in accuracy from the use of higher order terms. 


\begin{tabular}{c|c|lll}
\multirow{2}{*}{$\mathrm{n}$} & Precise & \multicolumn{3}{|c}{ Approximations (rounded to nearest integer) } \\
\cline { 3 - 5 } & $\begin{array}{c}\text { value } \\
\text { of } t_{n}\end{array}$ & $\begin{array}{l}\text { First } \\
\text { order }\end{array}$ & $\begin{array}{l}\text { Second } \\
\text { order }\end{array}$ & $\begin{array}{l}\text { Third } \\
\text { order }\end{array}$ \\
\hline 18 & 123867 & 115396 & 121213 & 122797
\end{tabular}

3.3. Special Types of Trees In [3] the authors study: $U_{n}$, the number of nonisomorphic identity rooted trees on $n$ points; $u_{n}$, the number of free identity trees on $n$ points; $H_{n}$, the number of nonisomorphic planted homeomorphically irreducible trees on $n$ points; $h_{n}$, the number of free homeomorphically irreducible trees of order $n ; C_{n}$, the number of nonisomorphic planted blocky trees on $n$ points; and $c_{n}$, the number of free blocky trees of order $n$. They use the generating function identities for $U(x), u(x), H(x), h(x), C(x)$, and $c(x)$ (formulae $(1),(2),(7),(8),(12)$, and (13) of [3]) to determine the radii of convergence of the generating functions and the first order terms in asymptotic expansions. ((1), (2), (7), and (8) are originally from [2].) Using Steps 1 through 4 for $U_{n}, H_{n}$, and $C_{n}$, and the procedure in Section 2.2 for $u_{n}, h_{n}$, and $c_{n}$, we obtained with computer algebra:

THEOREM 3. (a) $U_{n}$ has the asymptotic expansion

$$
\mu^{-n} n^{3 / 2}\left(0.3625364234-\frac{0.1044426616}{n}-\frac{0.2410458974}{n^{2}}-\frac{1.272504538}{n^{3}}+\ldots\right),
$$

and $u_{n}$ has the asymptotic expansion

$$
\mu^{-n} n^{-5 / 2}\left(0.2993882875-\frac{0.3488387859}{n}-\frac{1.335147424}{n^{2}}+\ldots\right),
$$

where $\mu=0.3972130969$.

(b) $H_{n}$ has the asymptotic expansion

$$
\theta^{-n} n^{-3 / 2}\left(0.4213018529+\frac{0.1320316948}{n}+\frac{0.6099539181}{n^{2}}+\frac{2.983030203}{n^{3}}+\ldots\right),
$$

and $h_{n}$ has the asymptotic expansion

$$
\theta^{-n} n^{-5 / 2}\left(0.6844472720+\frac{1.863425043}{n}+\frac{11.23522366}{n^{2}}+\ldots\right),
$$

where $\theta=0.4567332096$.

(c) $C_{n}$ has the asymptotic expansion

$$
\gamma^{-n} n^{-3 / 2}\left(0.3687229874-\frac{0.02892358469}{n}+\frac{0.05998637958}{n^{2}}+\frac{0.2476468907}{n^{3}}+\ldots\right),
$$


and $c_{n}$ has the asymptotic expansion

$$
\gamma^{-n} n^{-5 / 2}\left(0.3149782093-\frac{0.033901824744}{n}+\frac{0.3502928548}{n^{2}}+\ldots\right)
$$

where $\gamma=0.2225111687$.

REMARK. The above theorem shows that [3] incorrectly computed $\mu$ by about $1 / 2 \%$, and incorrectly computed the coefficients of all first order terms.

Here is some numerical evidence of the improvement in accuracy from the use of higher order terms.

\begin{tabular}{|c|c|c|c|c|c|}
\hline \multirow[b]{2}{*}{$\mathbf{n}$} & \multirow{2}{*}{$\begin{array}{c}\text { Precise } \\
\text { value } \\
\text { of } U_{n}\end{array}$} & \multicolumn{4}{|c|}{ Approximations (rounded to nearest integer) } \\
\hline & & $\begin{array}{l}\text { First } \\
\text { order }\end{array}$ & $\begin{array}{l}\text { Second } \\
\text { order }\end{array}$ & $\begin{array}{l}\text { Third } \\
\text { order }\end{array}$ & $\begin{array}{l}\text { Fourth } \\
\text { order }\end{array}$ \\
\hline 20 & 416848 & 423970 & 417863 & 417159 & 416973 \\
\hline & Precise & \multicolumn{4}{|c|}{ Approximations (rounded to nearest integer) } \\
\hline $\mathrm{n}$ & $\begin{array}{l}\text { value } \\
\text { of } u_{n}\end{array}$ & $\begin{array}{l}\text { First } \\
\text { order }\end{array}$ & $\begin{array}{l}\text { Second } \\
\text { order }\end{array}$ & $\begin{array}{l}\text { Third } \\
\text { order }\end{array}$ & \\
\hline 20 & 16104 & 17506 & 16486 & 16291 & \\
\hline
\end{tabular}

\begin{tabular}{c|c|cccl}
\multirow{2}{*}{$\begin{array}{c}\text { Precise } \\
\text { value }\end{array}$} & \multicolumn{4}{|c|}{ Approximations (rounded to nearest integer) } \\
\cline { 3 - 6 } & of $H_{n}$ & $\begin{array}{l}\text { First } \\
\text { order }\end{array}$ & $\begin{array}{l}\text { Second } \\
\text { order }\end{array}$ & $\begin{array}{l}\text { Third } \\
\text { order }\end{array}$ & $\begin{array}{l}\text { Fourth } \\
\text { order }\end{array}$ \\
\hline 20 & 30802 & 30185 & 30658 & 30768 & 30794
\end{tabular}

\begin{tabular}{c|c|ccc}
\multirow{2}{*}{$n$} & Precise & \multicolumn{3}{|c}{ Approximations (rounded to nearest integer) } \\
\cline { 3 - 5 } value & of $h_{n}$ & $\begin{array}{l}\text { First } \\
\text { order }\end{array}$ & $\begin{array}{l}\text { Second } \\
\text { order }\end{array}$ & $\begin{array}{l}\text { Third } \\
\text { order }\end{array}$ \\
\hline 20 & 2988 & 2452 & 2786 & 2886
\end{tabular}

\begin{tabular}{c|c|llll}
\multirow{2}{*}{$\mathrm{n}$} & Precise & \multicolumn{4}{|c}{ Approximations (rounded to nearest integer) } \\
\cline { 3 - 6 } $\mathrm{nalue}$ & of $C_{n}$ & $\begin{array}{l}\text { First } \\
\text { order }\end{array}$ & $\begin{array}{l}\text { Second } \\
\text { order }\end{array}$ & $\begin{array}{l}\text { Third } \\
\text { order }\end{array}$ & $\begin{array}{l}\text { Fourth } \\
\text { order }\end{array}$ \\
\hline 10 & 38982 & 39191 & 38883 & 38947 & 38973
\end{tabular}

\begin{tabular}{|c|c|c|c|c|}
\hline \multirow[b]{2}{*}{ n } & \multirow{2}{*}{$\begin{array}{l}\text { Precise } \\
\text { value } \\
\text { of } c_{n}\end{array}$} & \multicolumn{3}{|c|}{ Approximations (rounded to nearest integer) } \\
\hline & & $\begin{array}{l}\text { First } \\
\text { order }\end{array}$ & $\begin{array}{l}\text { Second } \\
\text { order }\end{array}$ & $\begin{array}{l}\text { Third } \\
\text { order }\end{array}$ \\
\hline 10 & 3424 & 3348 & 3312 & 3349 \\
\hline
\end{tabular}


With the exception of $c_{n}$ we see substantial improvement as the order of approximation is increased. For $c_{n}$ the following holds. By $n=20$ the first order approximation is larger than the precise value; by $n=30$ the second order approximation is a slight improvement on the first order approximation; by $n=60$ the second order approximation is a significant improvement on the first order approximation; and in all these cases the third order approximation is significantly better than the second order approximation.

\section{References}

[1] E. A. Bender, 'Asymptotic methods in enumeration', Siam Rev. 16 (1974), 485-515.

[2] F. Harary and G. Prins, 'The number of homeomorphically irreducible trees and other species’, Acta Math. 101 (1959), 141-162.

[3] F. Harary, R. W. Robinson and A. J. Schwenk, 'Twenty step algorithm for determining the asymptotic number of trees of various species', J. Austral. Math. Soc. (Series A) 20 (1975), 483-503.

[4] L. Hormander, An introduction to complex analysis in several variables (Van Nostrand, Princeton, 1966).

[5] R. Otter, 'The number of trees', Ann. of Math. 49 (1948), 583-599.

[6] J. M. Plotkin and J. W. Rosenthal, 'Some asymptotic methods in combinatorics', J. Austral. Math. Soc. (Series A) 28 (1979), 452-460.

[7] - 'The expected complexity of analytic tableaux analyses in propositional calculus', Notre Dame J. of Formal Logic 23 (1982), 409-426.

[8] G. Pólya, 'Kombinatorische Anzahlbestimmungen für Gruppen, Graphen und Chemische Verebindungen', Acta Math. 86 (1937), 145-254.

[9] G. Szegö, Orthogonal Polynomials, Colloquium Publications 23, revised edition (Amer. Math. Soc., Providence, 1959).

[10] R. J. Walker, Algebraic curves (Princeton University Press, Providence, 1978).

Department of Mathematics

Michigan State University

East Lansing, Michigan 48824
Department of Mathematics and Computer Science Ithaca College

Ithaca, New York 14850 